\title{
Beat Phenomenon in Metal Nanowires: A Molecular Dynamics
}

\section{Study}

Zhuoqun Zheng ${ }^{1}$, Eric $\mathrm{Li}^{2}$, Nan $\mathrm{Ding}^{1}, \mathrm{Xu} \mathrm{Xu}^{1^{*}}$

${ }^{1}$ College of Mathematics, Jilin University, 2699 Qianjin Street, Changchun, 130012, China

${ }^{2}$ Department of Mechanical and Automation Engineering, The Chinese University of Hong Kong, Hong Kong, China

\begin{abstract}
In this paper, a special beat phenomenon of metal nanowires is investigated by using the large scale molecular dynamics simulations. It is observed that the beat phenomenon exists not only in the vibration of $\langle 110\rangle$ orientated Face Center Cubic (FCC) nanowires of different materials, but also in the vibration of $\langle 100\rangle$ orientated Body Center Cubic (BCC) nanowires. Based on the atomic arrangement, a discrete spring-mass model is developed to explain the displacement characteristics of nanowire's vibration. It is found that the vibration frequency of nanowires rises slightly with the increase of initial actuation amplitude. The displacements of a typical atom in the nanowire are used to show the dynamical characteristics of beat phenomenon in vibration experiments. In addition, the beat phenomenon driven by a single actuation along one of the elementary directions has also been observed and shown in this work. Furthermore, a theoretical analysis is given for the excitation mechanism of beat phenomenon by analyzing the relation of excitation frequency between the two elementary directions.
\end{abstract}

Keywords: beat phenomenon, metal nanowires, molecular dynamics, elementary directions, vibration, excitation frequency.

\section{Introduction}

With the rapid progress of nano science and technology, theory and applications of the nanowires have been widely explored in many fields of science and engineering. Driven by their impressive electronic, thermal, mechanical and optical properties, nanowires have been widely used as active components of nanoelectromechanical systems (NEMS) such as force and pressure sensing [1, 2], nanowire-nanopore sensors [3], field effect transistor [4], lithium battery anodes [5] and other devices [6-8]. Among various nanowires, $\mathrm{BCC}$ Fe nanowires are

\footnotetext{
* Corresponding author: xuxu@jlu.edu.cn
} 
widely used in data storage and memory devices, spin electronics and smart sensors due to their excellent magnetic properties [9-13]. The NEMS utilize the nanowire as a resonating beam, in which the nanowire vibrates continuously at or near its resonant frequency. In addition, the changes in local environment including force, pressure or mass can be detected by the corresponding changes in the resonance frequency of the nanowire [14]. Therefore, it is of great significance to study the nanowire's mechanical properties under vibration.

In the past few years, there have been a large number of studies on the nanowire's vibrational properties, including the experimental studies [15-18], theoretical analysis [19-21] and computational modelling [22-24]. Olsson et al. studied the resonant properties of unstressed and prestressed gold nanowires and compared MD simulations' results with Euler Bernoulli beam theory and Timoshenko beam theory [25]. Two-dimensional vibration has been studied by Conley et al. theoretically. They explored the nonlinear dynamics of resonators and demonstrated that they can suddenly transit from a planar motion to a whirling, “jump rope" like motion [19]. Gil-Santos et al. proposed a new approach to mass sensing and stiffness spectroscopy based on the fact that the nanoresonator will enter a superposition state of two orthogonal vibrations with different frequencies when the symmetry is broken [6].

Beat phenomenon is a special physical characteristic in the vibration theory. A combination of two simple harmonic vibrations with almost same frequency can generate a periodic pulsation trend in energy and displacement amplitude of the vibration, namely the beat phenomenon [22]. Compared with the nanowires' mechanical properties, few studies have focused on the beat phenomenon because the continuum mechanics-based models do not capture the discrete lattice properties that are required to induce the beat phenomena. It is reported that for the beat phenomenon, there are two orthogonal elementary orientations in $<110>$ orientated FCC metal nanowires [23]. However, the mechanism of beat phenomenon is still not clear. Additionally, in most papers, only energy time history has been used to show the properties of beat phenomenon. Are there any other characteristics that can be used to study the beat phenomenon? The displacement is such a characteristic. It is quite important for the study of the nanowires' vibrational properties. However, little attention has been paid to this characteristic. 
Therefore, a further numerical and theoretical study of beat phenomenon is presented in this work. We analyze the beat phenomenon on $\langle 100\rangle$ orientated BCC Fe nanowires, which remains valid for this phenomenon. Based on the atomic structure, a valid discrete spring-mass model has been proposed to show the lattice arrangement properties and study the vibrations of the nanowire. With the results of Molecular Dynamics (MD) simulations and the spring-mass model, the vibration frequency of nanowires is found to increase slightly with the rise of initial actuation amplitude. As the displacement characteristic is a significant property, we investigate it by studying the displacement time history of a typical atom, which is selected from the nanowire. The displacement time histories of the MD simulations reveal that the vibrations in the orthogonal elementary directions have an influence on each other. In addition, we find that there exists the beat phenomenon driven by a single actuation along one of the elementary directions, which is distinctly shown with the displacement characteristic by our method. By further study, we also give a reasonable excitation mechanism of the beat phenomenon.

\section{Models and Methods}

\section{Atomistic Simulation}

In this work, the vibration tests of double clamped Fe nanowires are performed by using the Large-scale Atomic/Molecular Massively Parallel Simulator (LAMMPS) [26]. The Fe nanowires used in simulation are $\langle 100\rangle$ orientated and created with atoms in positions corresponding to a bulk perfect BCC crystal lattice. Fig. 1 shows the model of <100> orientated Fe nanowires. In this model, the shape of cross-section is chosen to be square. Atoms at both ends of the nanowires are fixed, and are denoted by "A" in Fig. 1; while the rest of the nanowires are free to move. The periodic boundary conditions are not imposed in any directions. 


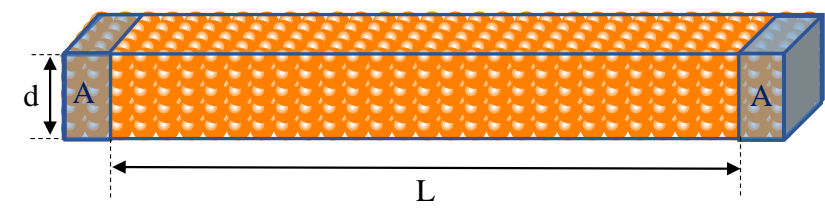

Figure 1. Schematic visualization of a fixed-fixed $\langle 100\rangle$ orientated Fe nanowire. The atoms in the areas ' $\mathrm{A}$ ' are fixed in all directions and the residual atoms can move freely.

The embedded-atom-method (EAM) potential developed by Mendelev et al [27] is used to describe the atomic interactions between $\mathrm{Fe}$ atoms in these simulations. It is a semi-empirical function fitted to a group of parameters, including elastic constants, equilibrium lattice constant, cohesive energy, unrelaxed vacancy formation energy, et al. In this model of atomic interaction, the total energy $E_{t o t}$ of a system with $N$ atoms is a sum of the classical pair potential and many-body embedding energy [28].

$$
E_{\text {tot }}=\sum_{i=1}^{N} F_{i}\left(\rho_{i}\right)+\sum_{i=1}^{N} \sum_{j=1}^{N} \phi_{i j}\left(R_{i j}\right)
$$

where $F, \rho, \phi \quad R_{i j}$ are the embedded energy, pair potential, electron cloud density and the distance between atom $i$ and $j$, respectively.

The centro-symmetry parameter (CSP) is used to recognize the plastic deformation, which is defined by [29]

$$
C S P=\sum_{i=1}^{4}\left|\mathbf{R}_{i}+\mathbf{R}_{i+4}\right|^{2}
$$

where $\mathbf{R}_{i}$ and $\mathbf{R}_{i+4}$ are vectors corresponding to the four pairs of opposite nearest neighbors in BCC lattice. The CSP value rises from zero for perfect BCC lattice to positive values for defects, and for atoms close to free surfaces.

At the beginning of each simulation, the nanowires are relaxed to the initial equilibrium configuration using the conjugate gradient energy minimization. Then, the Nose-Hoover thermostat $[30,31]$ is employed to equilibrate the nanowires at $0.2 \mathrm{~K}$. Finally, an initial velocity excitation $\mathbf{v}(z)$ is imposed on the nanowires along the $z$-axis as follows

$$
\mathbf{v}(z)=\left[A_{1} \sin \left(\frac{k_{1} \pi z}{L}\right), A_{2} \sin \left(\frac{k_{2} \pi z}{L}\right)\right]^{\mathrm{T}} \quad\left(k_{1}, k_{2}=1,2, \cdots\right)
$$

where, the subscripts 1 and 2 refer to the $x$ and $y$-axis. $A$ is actuation amplitude and $L$ is the effective length of the nanowires that exclude the two fixed edges. 


\section{Analysis Methods}

External energy is defined as the difference between the potential energy before and after the transverse velocity actuation is applied to the nanowire [22]. The energy data can be directly obtained from the simulations results, and the vibration frequency and vibration modes of the nanowire can be obtained by using the Fast Fourier transform (FFT).

The displacement of the nanowire is another important characteristic to show the mechanical properties of the nanowire, especially for the two-dimensional vibration. It is more intuitive to explore and show the properties of beat phenomenon by nanowire's displacement characteristic. However, there are few studies focused on the displacement characteristic of the two-dimensional vibrational nanowire due to the difficulties in collecting displacement data of the nanowire from the simulations. In this study, an atom of middle cross-sectional (001) plane of the $\mathrm{Fe}$ nanowire is selected as a sample to study the time history of displacement. The process is shown in Fig. 2.

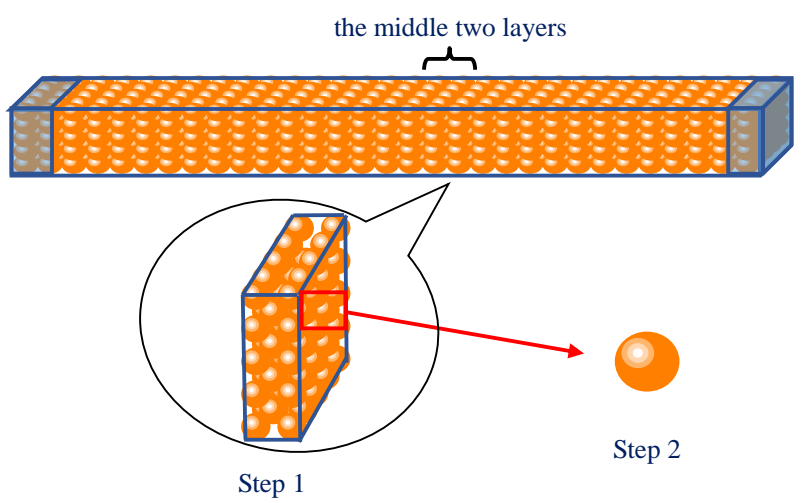

Figure 2. Schematic visualization of the process of choosing a typical atom from $\langle 100\rangle$ orientated $\mathrm{Fe}$ nanowire. Firstly, we take out the middle cross-sectional plane from the $\langle 100\rangle$ orientated Fe nanowire. Then, we choose an atom from the plane randomly because atoms on the plane are similar to each other in displacement.

\section{A Discrete Spring-mass Model}

From the view of lattice structure, a simplified discrete model is developed to explore the effects of nanowires' atomic structure on the vibrations. In the atomic arrangement of the $<100>$ orientated $\mathrm{Fe}$ nanowire, each internal lattice is surrounded by other six lattices. However, the two lattices along the $z$-axis have little effect on the displacements of atoms in the intermediate lattice because of the fixed end in the $z$-axis. Thus, we neglect the lattices 
along the $z$-axis. Besides, the lattices along the $x$-axis and $y$-axis have the same influence on the internal lattice. Fig. 3a shows the lattice structure.
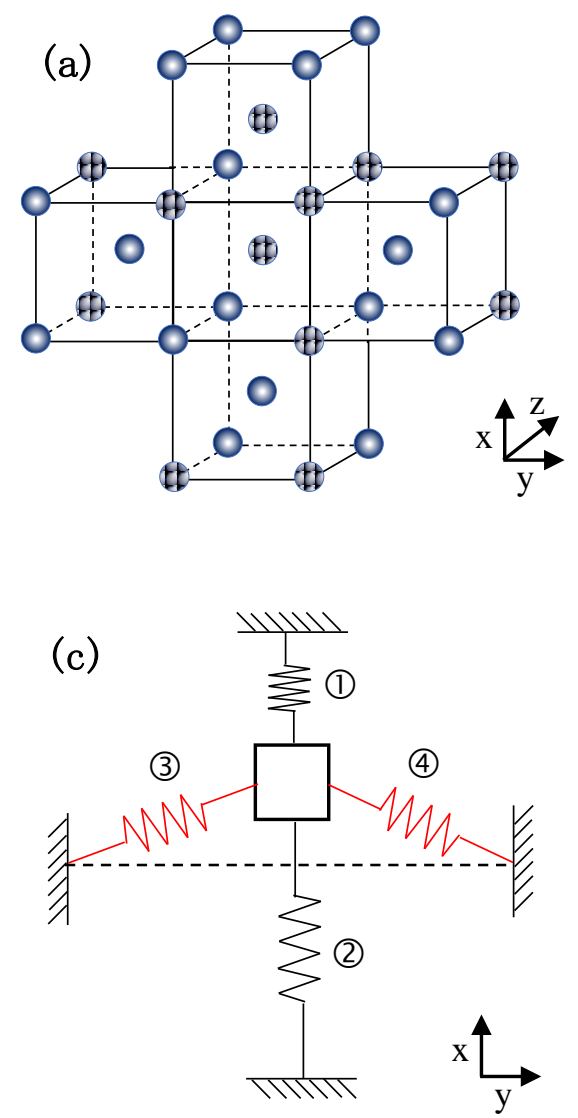
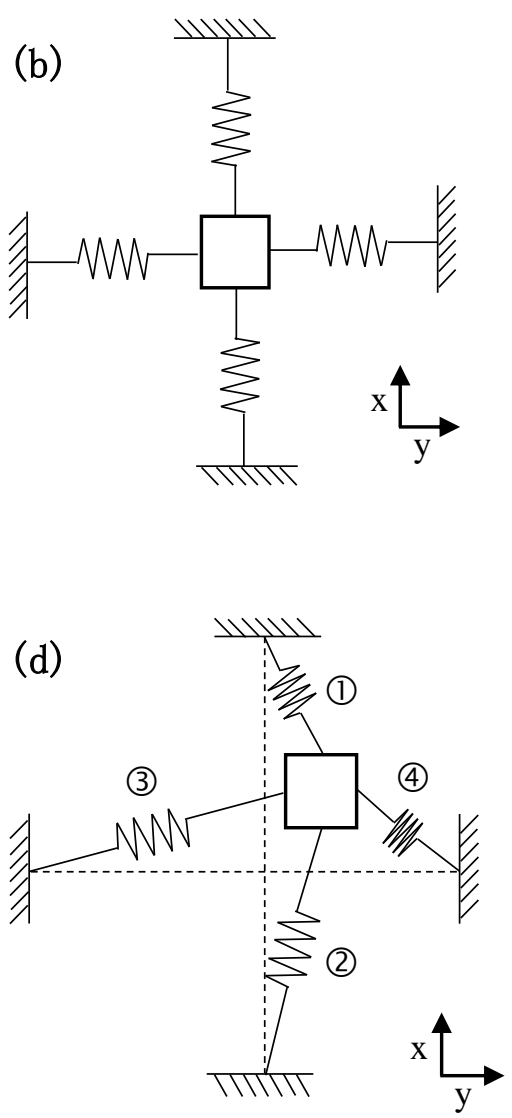

Figure 3. A discrete spring-mass model: (a) The typical atomic arrangement of $\mathrm{Fe}$ nanowires with several lattices. (b) Schematic of simplified discrete spring-mass model. (c) Schematic of spring-mass model in one-dimensional vibration. (d) Schematic of spring-mass model in two-dimensional vibration.

For simplicity, we simplify this structure as a spring-mass system as illustrated in Fig. 3b, and consider the vibrations of one-dimensional (Fig. 3c) and two-dimensional (Fig. 3d) models. Considering the geometric nonlinearity, the dynamic equations are described as one-dimensional vibration:

$$
m \ddot{x}=-4 k x+2 k x \frac{l_{0}}{\sqrt{x^{2}+l_{0}^{2}}}
$$

Two-dimensional vibration:

$$
\left\{\begin{array}{l}
m \ddot{x}=-4 k x-k l_{0}\left(\frac{-x}{\sqrt{x^{2}+\left(y-l_{0}\right)^{2}}}+\frac{-x}{\sqrt{x^{2}+\left(y+l_{0}\right)^{2}}}+\frac{-l_{0}-x}{\sqrt{\left(x+l_{0}\right)^{2}+y^{2}}}+\frac{l_{0}-x}{\sqrt{\left(x-l_{0}\right)^{2}+y^{2}}}\right) \\
m \ddot{y}=-4 k y-k l_{0}\left(\frac{l_{0}-y}{\sqrt{x^{2}+\left(y-l_{0}\right)^{2}}}+\frac{-l_{0}-y}{\sqrt{x^{2}+\left(y+l_{0}\right)^{2}}}+\frac{-y}{\sqrt{\left(x+l_{0}\right)^{2}+y^{2}}}+\frac{-y}{\sqrt{\left(x-l_{0}\right)^{2}+y^{2}}}\right)
\end{array}\right.
$$


where $m$ is mass of the object, $k$ and $l_{0}$ are elastic constant and initial length of the spring. The details on equations (4) and (5) can be referred to Appendix.

The spring-mass system we developed in this section is used to investigate the properties of nanowires' vibrations based on the atomic structure. Because of the simplicity, this model cannot substitute the nanowires in MD simulation. Therefore, only comparative analysis of phenomenon between this model and the nanowires is shown in this paper.

\section{Results and Discussion}

\subsection{One-dimensional vibration}

We begin this paper with the one-dimensional vibration simulations carried out on the $<100>$ orientated $\mathrm{Fe}$ nanowires. In order to generate the one-dimensional vibration, the initial velocity excitation is exerted on $x$-axis as follows.

$$
v(z)=A \sin \left(\frac{\pi z}{L}\right)(n m / s)
$$

The simulation is performed on the nanowire with the size of $5.74 \times 5.74 \times 28.7 \mathrm{~nm}^{3}$ and $A$ is chosen to be $0.15 \mathrm{~nm}$ in Eq. 6.The whole simulation lasts about 4000 picoseconds at a time step of 1 femtoseconds. Fig. 4a depicts the time history of the external energy during the free vibration after the initial velocity actuation. Fig. 4b presents part of the periodogram regarding the power of the discrete Fourier transformation versus frequency. As seen in Fig. $4 \mathrm{~b}$, main frequency component is identified as about $54.78 \mathrm{GHz}$. This result indicates that the Fe nanowire is under a vibration with the frequency of $27.39 \mathrm{GHz}$, because the frequency of the external energy is twice that of the actual vibration [22].
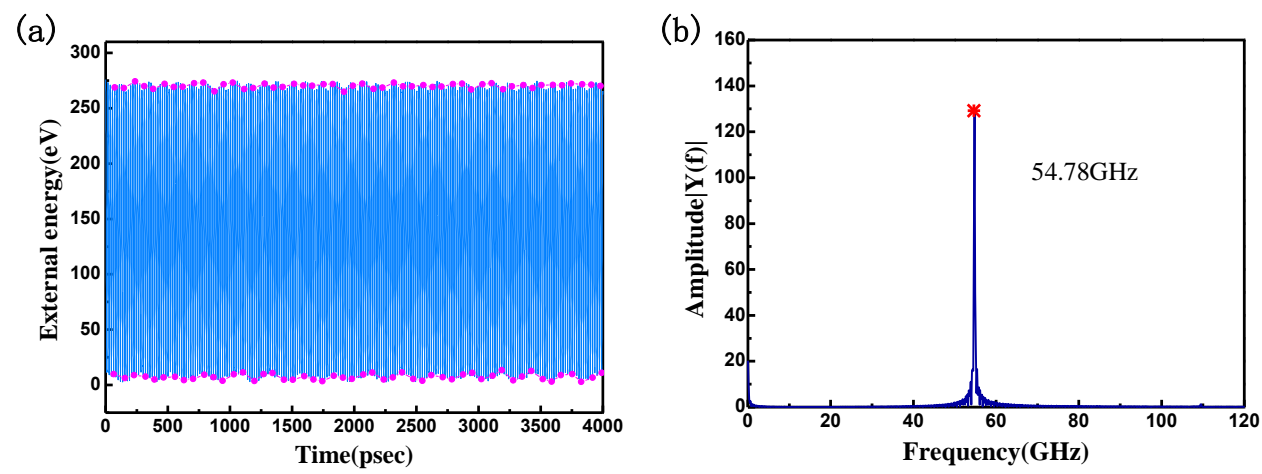

Figure 4. Free vibration MD simulation of $\mathrm{Fe}$ nanowire with the size of $5.74 \times 5.74 \times 28.7 \mathrm{~nm}^{3}$ : (a) 
External energy time history. Circle markers highlight the maximum and minimum of external energy during each vibration circle. (b) The frequency spectrum (from 0 to $120 \mathrm{GHz}$ ). The star marker highlights the maximum of the main frequency component.

Fe nanowires with different sizes under the same initial actuation amplitude are tested in the similar way. Nanowires with fixed cross section width to length ratio of 1:5 are utilized in this part, such as the sizes of $1.148 \times 1.148 \times 5.74 \mathrm{~nm}^{3}, 1.722 \times 1.722 \times 8.61 \mathrm{~nm}^{3}$, $2.87 \times 2.87 \times 14.35 \mathrm{~nm}^{3}$ and $5.74 \times 5.74 \times 28.7 \mathrm{~nm}^{3}$. The initial actuation amplitude is fixed at $0.15 \mathrm{~nm}$. Fig. 5 shows the vibration frequencies of nanowires with different sizes under the same initial actuation. It is easy to see that the vibration frequency descends obviously with an increase of cross-sectional width. Thus, it is clear that the vibration frequency is affected by the size of the nanowire in the nano scale, which is called size effect.

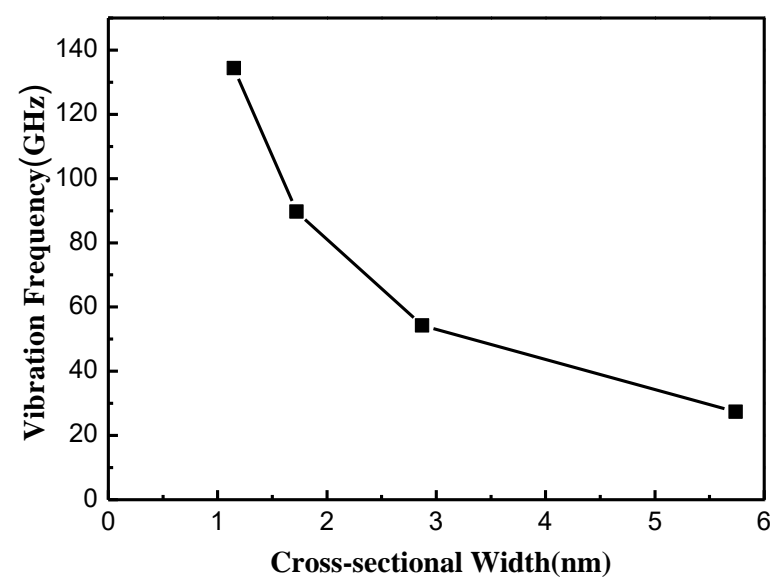

Figure 5. The vibration frequencies vs cross-sectional width under the same initial actuation.

Nanowires with the size of $1.148 \times 1.148 \times 5.74 \mathrm{~nm}^{3}$ and $1.722 \times 1.722 \times 8.61 \mathrm{~nm}^{3}$ are chosen for further simulations. They are tested under different initial actuation amplitudes, ranging from $0.02 \mathrm{~nm}$ to $0.14 \mathrm{~nm}$. Fig. 6a describes the frequencies obtained from FFT analysis. It is found that the vibration frequency from MD simulations rises slightly with the increase of initial actuation amplitude. However, according to the classical theory, the Euler-Bernoulli beams have fixed natural frequencies, which is irrelevant to initial actuation amplitude. In addition, we test the vibration frequencies on the spring-mass model using Eq. 4 with different actuation amplitudes. Fig. $6 \mathrm{~b}$ shows that the vibration frequency rises slowly with the increase of initial actuation amplitude. The results are similar to those from MD simulations. 

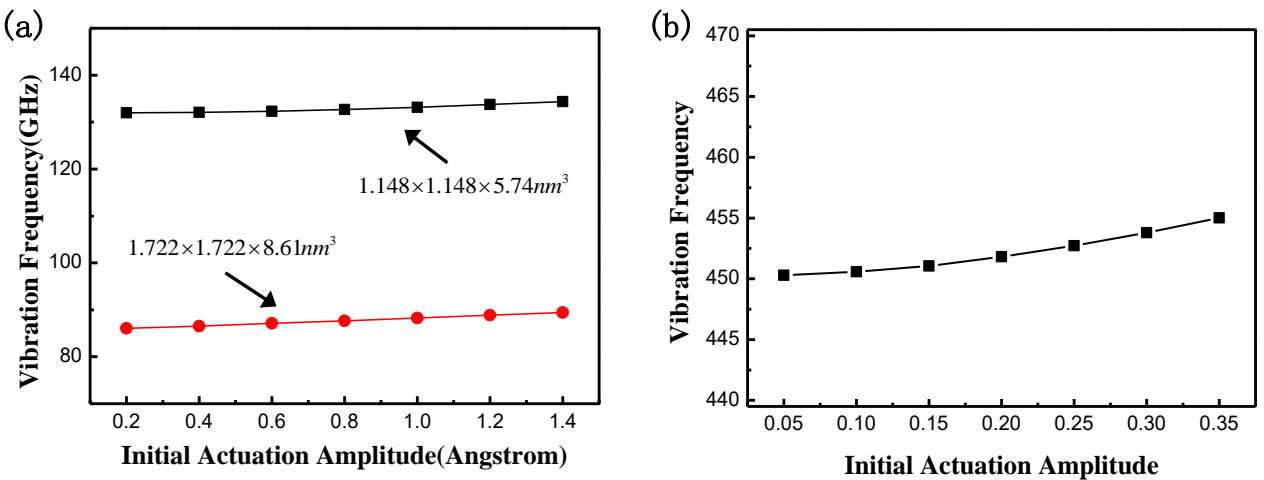

Figure 6. Vibration frequencies vs initial actuation amplitude: (a) nanowires with the size of $1.148 \times 1.148 \times 5.74 \mathrm{~nm}^{3}$ and $1.722 \times 1.722 \times 8.61 \mathrm{~nm}^{3}$. (b) spring-mass model.

It is easy to see that the results from MD simulations are consistent with those from the spring-mass model based on atomic structure, instead of the classical Euler-Bernoulli theory. We suggest that, in the nano scale, the lattice structure has a big influence on the properties of the nanowires' vibrations. As mentioned above, according to the atomic arrangement, the spring-mass model has geometric nonlinearity, which may result in the positive correlation between vibration frequency and initial actuation amplitude. However, the classical Euler-Bernoulli model is a macro-continuity model, which may be not suitable for the nanowire in this scale. Therefore, in this case, it is found that the vibration frequency increases slightly with the growth of initial actuation amplitude.

\subsection{Beat Phenomenon}

In this section, $<100>$ orientated Fe nanowire with the size of $1.148 \times 1.148 \times 5.74 \mathrm{~nm}^{3}$ is used to exhibit the beat phenomena using the MD simulation. The initial velocity excitation on $x$-axis and $y$-axis for generating beat phenomenon is expressed as follows

$$
\mathbf{v}(z)=\left[0.05 \sin \left(\frac{\pi z}{5.74}\right), 0.15 \sin \left(\frac{\pi z}{5.74}\right)\right]^{\mathrm{T}}
$$

Fig. 7a depicts the time history of the external energy during the free vibration after exerting the initial velocity actuation. Fig. $7 \mathrm{~b}$ presents the periodogram regarding the power of the discrete Fourier transformation versus frequency. As a special physical characteristic in two-dimensional vibration, beat phenomenon can be identified when the external energy amplitude exhibits a periodic pulsation pattern [23]. It is clearly seen from Fig. 7a that a periodic pulsation occurs, while two frequency components are identified as $267.49 \mathrm{GHz}$ and 
271.00 GHz in Fig. 7b. Their amplitudes are observably larger than other frequency components in the frequency spectrum from FFT analysis. Therefore, it can be concluded that the Fe nanowire admit a beat vibration state. In addition, since the vibration frequencies of the external energy is twice the vibration frequencies of the displacement, the actual frequencies of the displacements are approximated $133.74 \mathrm{GHz}$ and $135.50 \mathrm{GHz}$. Fig. $7 \mathrm{c}$ shows the energy time history of the spring-mass model. We can easily find that this curve has a periodic pulsation pattern in amplitude, which is similar to the curve from the MD simulation.
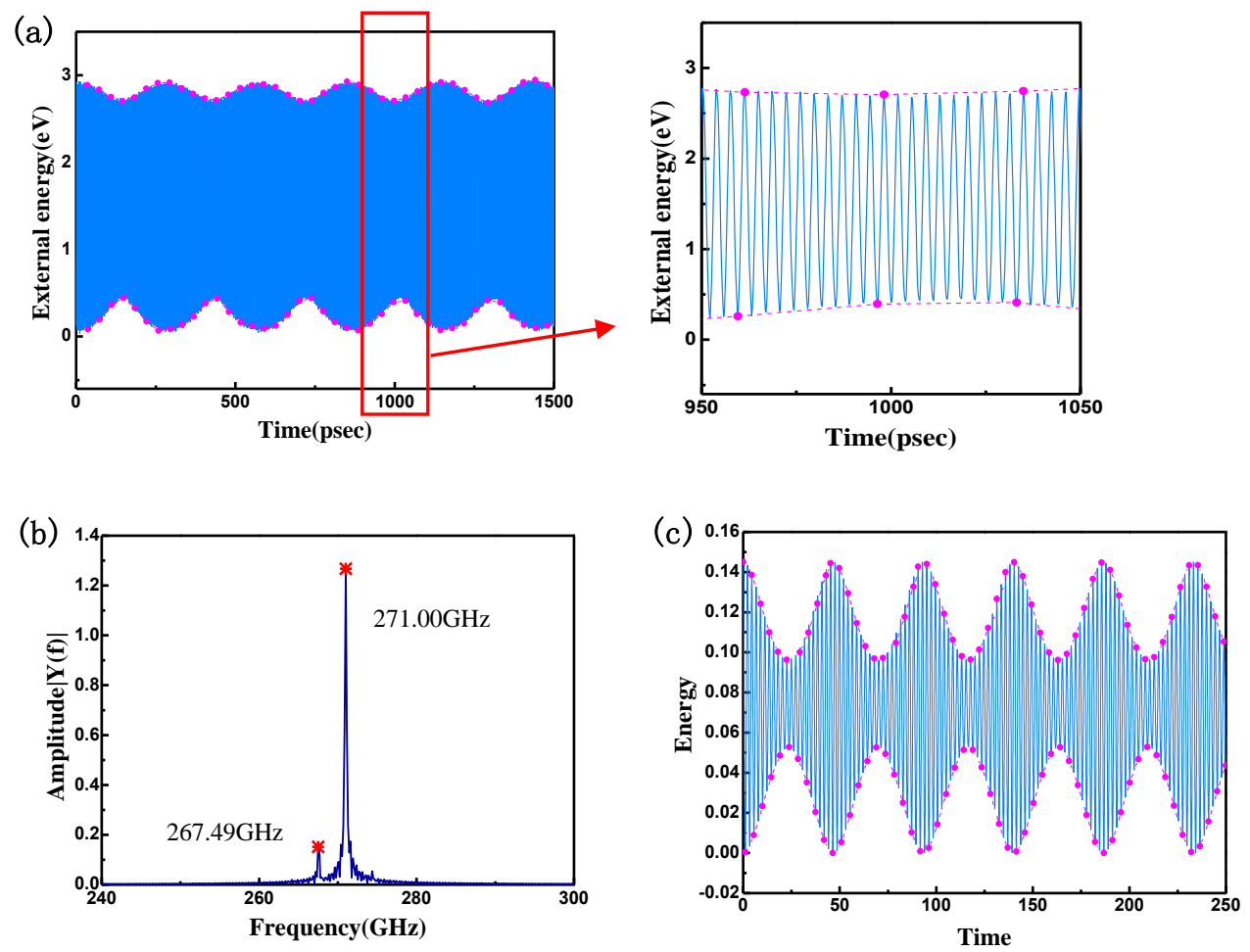

Figure 7. The results of a free vibration MD simulation carried on the $\langle 100\rangle$ orientated Fe nanowire with the size of $1.148 \times 1.148 \times 6.888 \mathrm{~nm}^{3}$. (a) external energy time histories. The left figure is from 0 to 1500 picosecond, while the right one from 950 to 1050 picosecond. (b) frequency spectrum from FFT analysis (from 240 to $300 \mathrm{GHz}$ ). (c) energy time history of the spring-mass model.

The video of the beat phenomenon in $\langle 100\rangle$ orientated Fe nanowire (see supplementary material) show the novel vibration in two orthogonal elementary directions clearly and intuitively.

In the following, we use the displacement characteristic of the nanowire to investigate the properties of beat phenomenon. As mentioned in Analysis Methods in Section 2, an atom of the Fe nanowire is selected as a sample to study the time history of displacement. Fig. 2 
shows the process of the selection. The displacement data of the typical atom have been collected from the MD simulations. Figs. $8 \mathrm{a}$ and $8 \mathrm{c}$ show the time history of displacements of the typical atom in the $x-z$ plane and the $y-z$ plane. Figs. $8 \mathrm{~b}$ and $8 \mathrm{~d}$ are their corresponding frequency spectrums from FFT analysis. The main frequencies of the Fe nanowire in the $x$-axis and $y$-axis shown in Figs. $8 \mathrm{~b}$ and $8 \mathrm{~d}$ are $133.82 \mathrm{GHz}$ and $135.50 \mathrm{GHz}$. These results agree with those in Fig. 7b, which indicates the effectiveness of the typical atom. It is clearly observed from Figs. $8 \mathrm{a}$ and $8 \mathrm{c}$ that both the amplitudes of the displacements in the $x$-axis and $y$-axis exhibit a periodic pulsation pattern. We also obtain the displacement data from numerical simulations of the spring-mass model, which are presented in Figs. 8e and 8f. Similarly, the periodic pulsation patterns have been found in the amplitudes of these displacement time histories. The similarity between the results of MD simulation and spring-mass model demonstrates that the atom structure plays an important role in the beat phenomenon. Further, this result can give an explanation to the existence of two orthogonal elementary orientations for the beat phenomenon in nanowires [23]. It is related to the lattice structure.
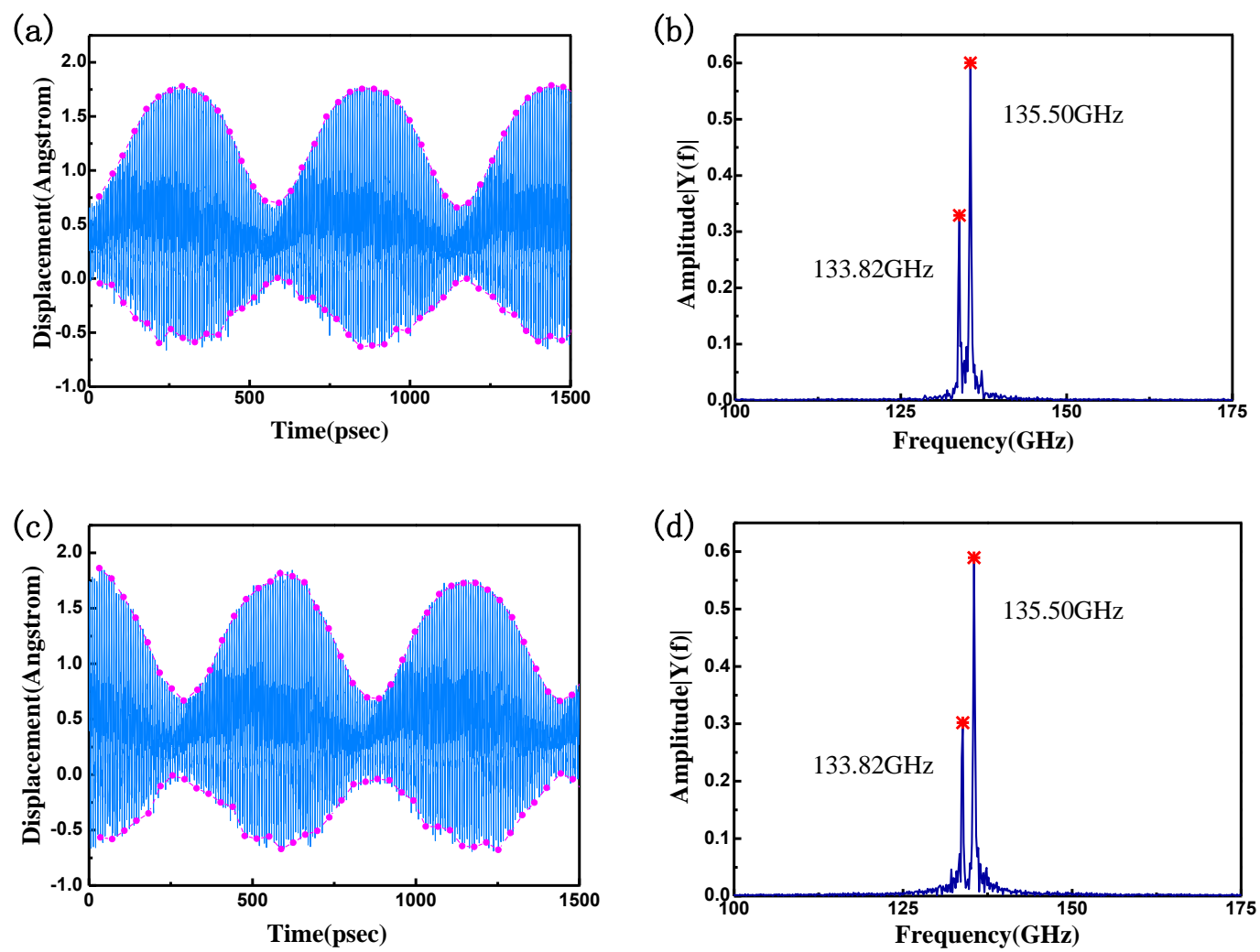

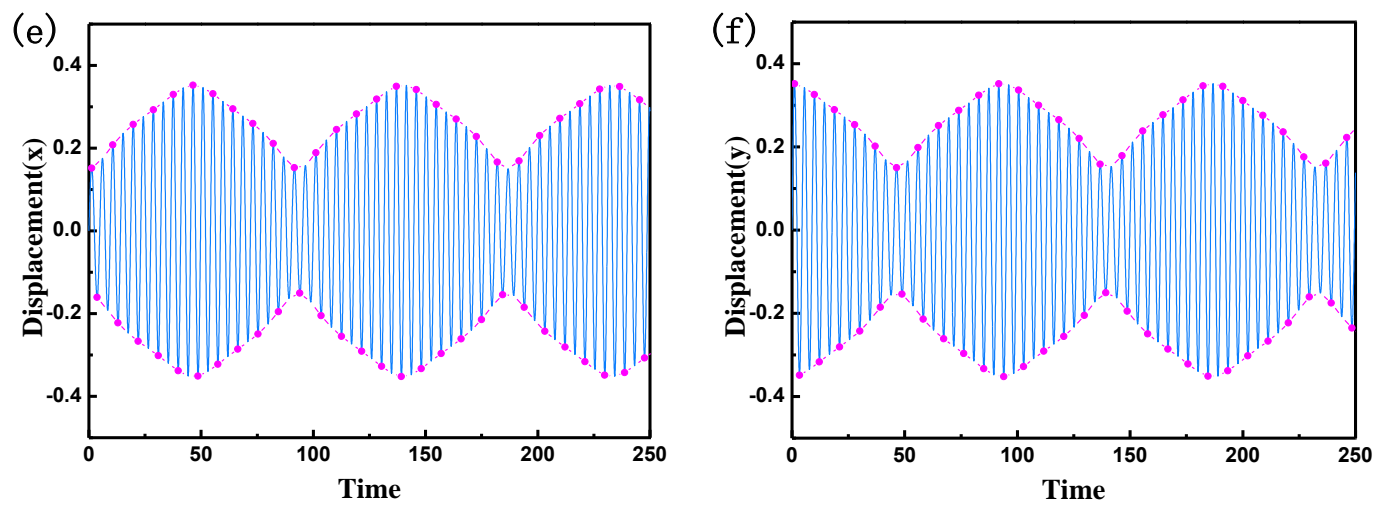

Figure 8. Displacement and corresponding frequency spectrums of the typical atom: (a) and (c) displacement time histories in $x$-axis and $y$-axis in the MD simulation; (b) and (d) corresponding frequency spectrums from FFT analysis (from 100 to $175 \mathrm{GHz}$ ); (e) and (f) displacement time histories in $x$-axis and $y$-axis from the spring-mass system.

In order to further study the displacement characteristics, we put the displacement time histories of the $x$-axis and $y$-axis in one figure (see Fig. 9a). From these figures, it is clear that the displacement in the $x$-axis is completely opposite to that in the $y$-axis. And the $x$-axis has the maximum amplitude of the displacement, when the $y$-axis has the minimum amplitude, and vice versa. These observations imply that during the beat vibration, the vibrations in the $x$-axis and $y$-axis interact with each other. In addition, as shown in Fig. 9b, we find the same phenomenon in the spring-mass system.
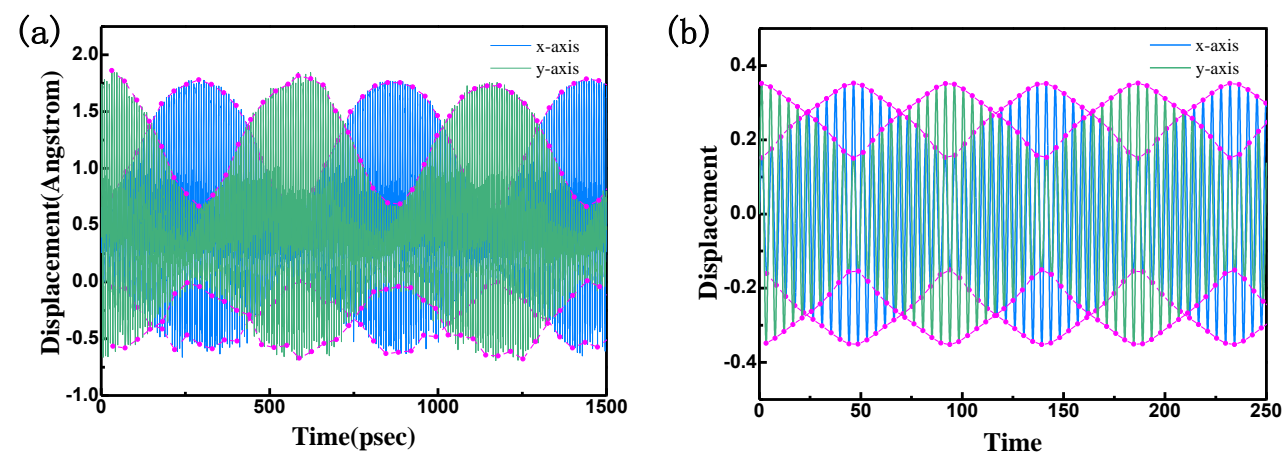

Figure 9. Combination of the displacements in the $\mathrm{x}$-axis and y-axis: (a) the typical atom from MD; (b) the spring-mass model.

\subsection{Elastic Region and Plastic Region}

Further interest is focused on the beat phenomenon driven by a single actuation along one of the elementary directions, which is the origin of beat phenomenon's finding [22]. In this section, the nanowires vibrate in vacuum without damping. These MD simulations are in NVE ensemble except the energy minimization at the beginning. Thus, the whole system is 
energy conservation. As we know, the plastic deformation of nanowires will consume energy. In an environment where there is no energy dissipation, the attenuation of the external energy's amplitude indicates that the vibration energy is converting into plastic deformation energy. Therefore, the attenuation of the external energy's amplitude can be used to recognize the plastic deformation that occurs in the vibration.

The $\langle 100\rangle$ orientated Fe nanowire with the size of $1.722 \times 1.722 \times 8.61 \mathrm{~nm}^{3}$ is tested. Firstly, initial velocity excitation with a small amplitude is exerted only in $x$-axis direction as follows:

$$
v(z)=0.1 \sin \left(\frac{\pi z}{8.61}\right)(n m / s)
$$

The external energy of the nanowire and displacements of a typical atom are shown in Fig. 10. In Fig. 10a, there is no attenuation in the energy time history, which means the vibration of the nanowire is still in the elastic state. The external energy amplitude does not exhibit a periodic pulsation pattern. Figs. 10b and 10c show that the nanowire only vibrates in the pre-set direction ( $x$-axis). These results indicate that there is no beat phenomenon in this case.
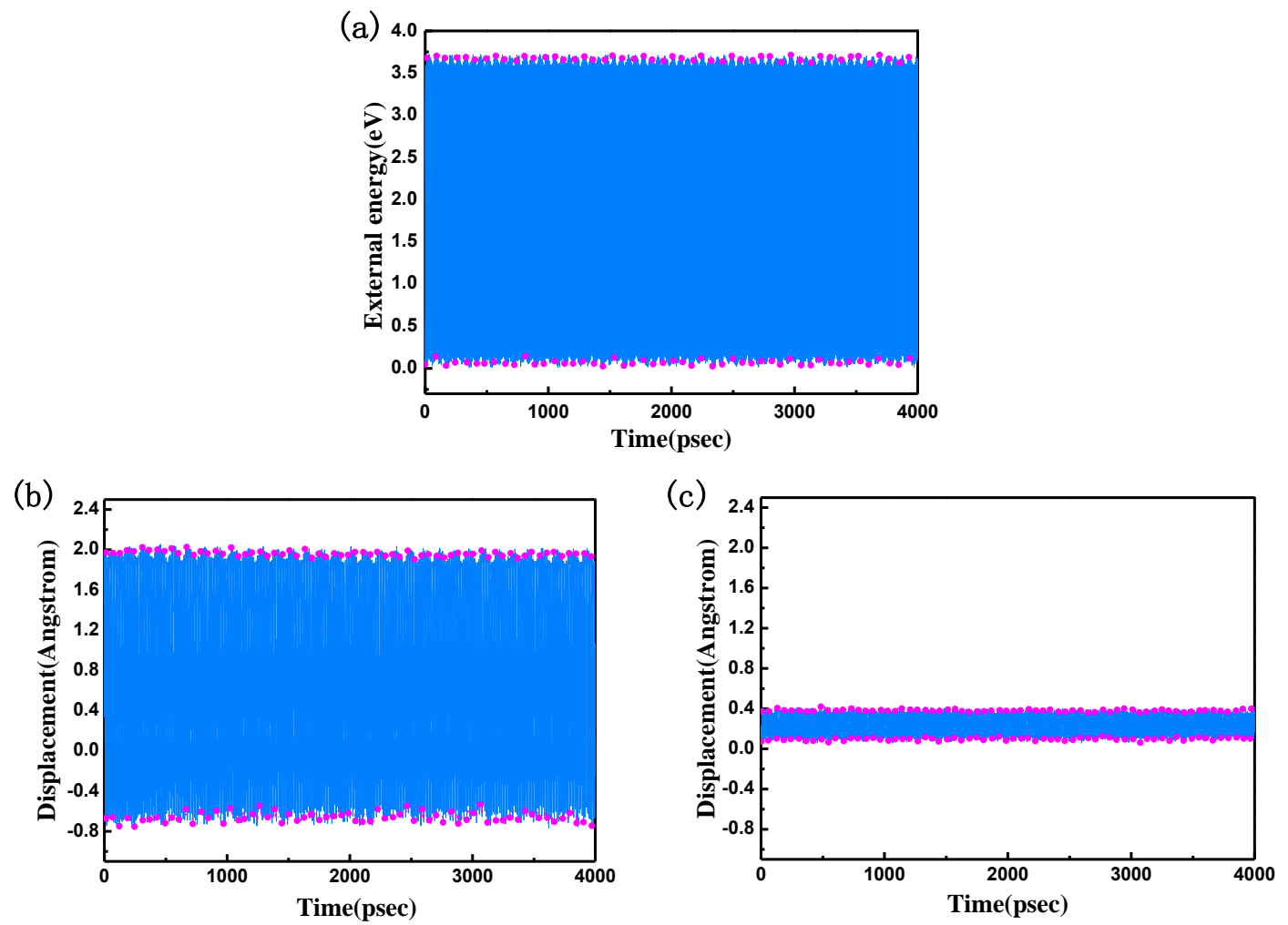

Figure 10. External energy of the nanowire and displacements of a typical atom for a small initial excitation: (a) the energy time history; (b) and (c) displacement time histories of a typical atom in $\mathrm{x}$-axis and $\mathrm{y}$-axis. No beat phenomenon in this case. 
Then, the large initial excitation amplitude 0.32 is exerted on the same nanowire only in $x$-axis direction as follows:

$$
v(z)=0.32 \sin \left(\frac{\pi z}{8.61}\right)(\mathrm{nm} / \mathrm{s})
$$

Fig. 11 presents the results of this simulation. As shown in Fig. 11a, there is an attenuation at the beginning of the external energy time history. When time comes to 2600 picosecond, it obviously exhibits the periodic pulsation pattern, which indicates the plastic deformation. Figs. $11 \mathrm{~b}$ and $11 \mathrm{c}$ describe the displacements in the $x$-axis and $y$-axis. At the beginning, the nanowire only vibrates in the $x$-axis. However, at about 2600 picosecond, both the amplitudes of the displacements in the $x$-axis and $y$-axis show the periodic pulsation pattern. These results imply that the beat phenomenon exists in this situation.
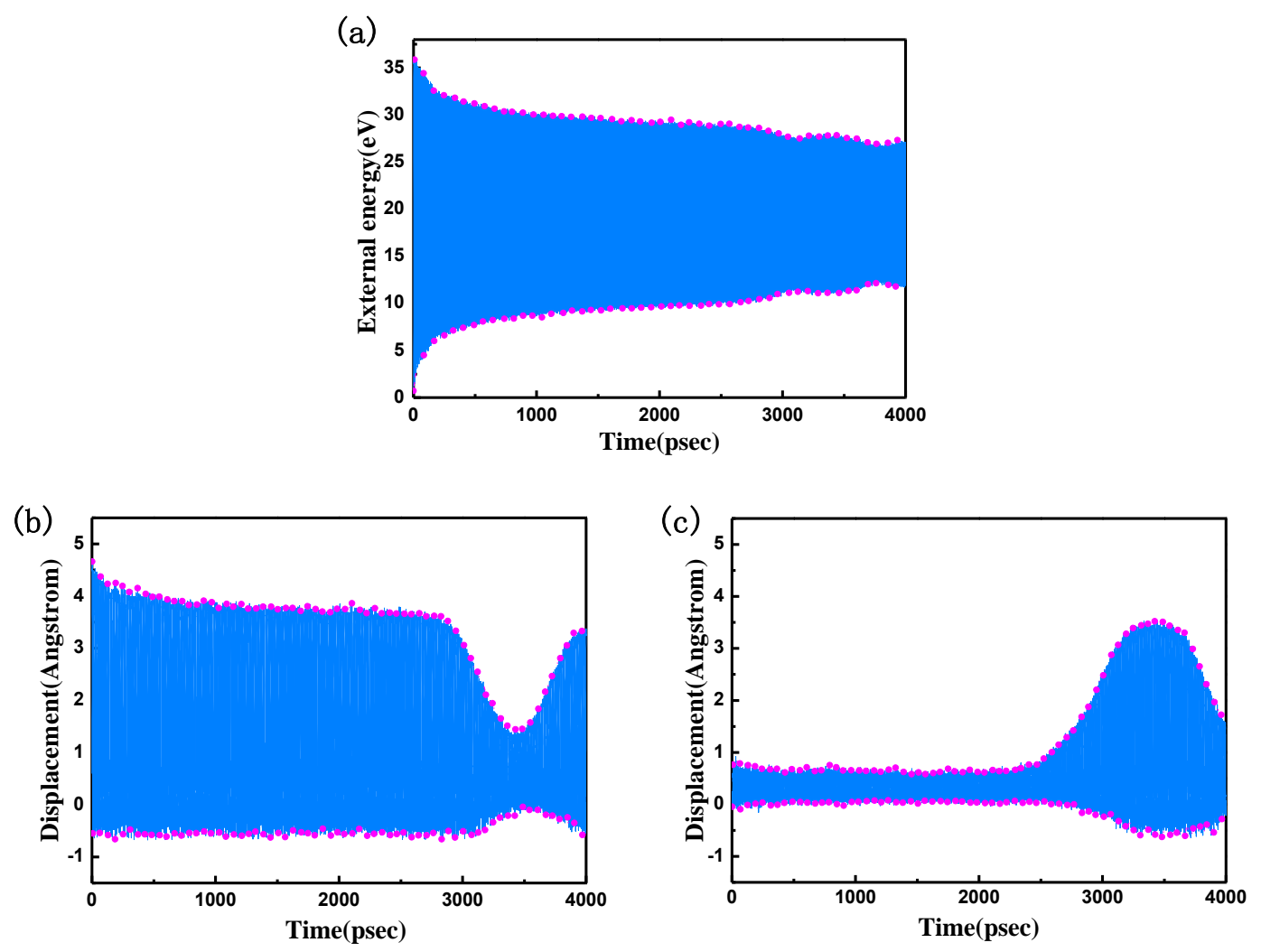

(d)

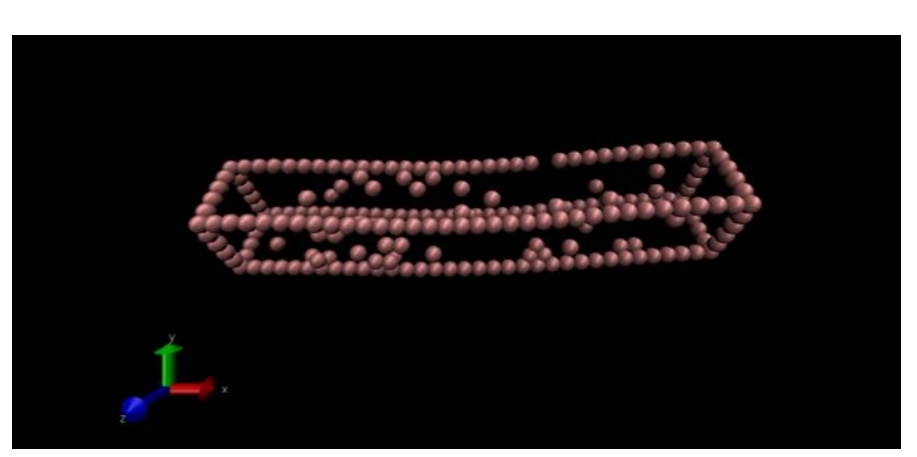


Figure 11. External energy of the nanowire and displacements of a typical atom for a large initial excitation: (a) the energy time history; (b) and (c) displacement time histories of a typical atom in $\mathrm{x}$-axis and y-axis; (d) the snapshot of atoms in the nanowire with CSP value between 0.1 and 7 at about 2600 picosecond. Beat phenomenon exists in this situation.

The attenuation in the external energy time history means the vibration is in the plastic region. The Fe nanowire begins to show the beat phenomenon at the time of 2600 picosecond in Fig.11a. By comparing the displacements of the typical atom in these two simulations (Fig. 10b, 10c and Fig. 11b, 11c), we can see that the beat phenomenon driven by the single initial velocity excitation. When the beat vibration occurs, the $y$-axis suddenly has an intense vibration at that time, which signifies that the vibration in $x$-axis decomposes into vibrations in $x$-axis and $y$-axis. The vibrations in $x$-axis and $y$-axis begin to affect each other. Therefore, when it comes to the plastic region, the vibration driven by the single initial velocity excitation may occur not only in the pre-set direction, but also in another elementary direction.

In Fig. 11d, atoms with CSP value between 0.1 and 7 are depicted. According to lattice constant and atomic structure, the CSP values ranging from 0.1 to 7 correspond to surfaces, partial dislocations and stacking faults [29]. Besides surface, we can see several partial dislocations in the nanowire. With the generation of partial dislocations, the crystal structure is no longer symmetric. In conclusion, the plastic deformation induces the decomposing of the vibration and this is necessary for a single actuation along one of the elementary directions to engender the beat phenomenon.

\subsection{Excitation Mechanism of Beat Phenomenon}

The beat phenomenon is usually generated by a combination of two vibrations with almost the same frequency in different directions. According to the classical beam theory, the natural frequency in each direction depends on the width and height of the nanowire. Here, the natural frequency is defined as the first order natural frequency of the nanowire. In the previous MD simulations, the cross section of the $<100>$ orientated Fe nanowire is chosen to be square, and thus the nanowire along $x$ and $y$ direction possesses the similar natural frequencies. In addition, the similar initial excitations are imposed on the nanowire in different directions, which induce similar excitation frequencies. Here, the excitation 
frequency is defined as the actual main vibration frequency of the nanowire. The results in MD simulations show that there exists the beat phenomenon in the nanowire with similar natural frequencies and excitation frequencies in different directions.

As mentioned by the previous researchers $[22,25]$, the high vibration modes could be excited respectively by the initial velocity excitation like this.

$$
v(z)=A \sin \left(\frac{k \pi z}{L}\right) \quad k=2,3, \cdots
$$

Thus, the nanowires with rectangle cross section actuated by different initial excitation forms in different directions can have similar natural frequencies, different excitation frequencies or similar excitation frequencies, different natural frequencies. Therefore, a question naturally arises: which frequencies (natural frequencies or excitation frequencies) can induce the beat phenomenon?

By a large number of simulation tests, it is found that the Fe nanowire with the size of $2.009 \times 0.574 \times 10.045 \mathrm{~nm}^{3}$ actuated by the initial velocity excitation separately $\left(v(z)=0.1 \sin \left(\frac{\pi z}{10.045}\right)(n m / s)\right.$ in the $x$-axis and $v(z)=0.1 \sin \left(\frac{2 \pi z}{10.045}\right)(n m / s)$ in the $y$-axis) has almost the same excitation frequencies as 70.80 and $74.31 \mathrm{GHz}$. The different natural frequencies such as 70.80 and $30.83 \mathrm{GHz}$ are also been identified in simulation tests when we apply these initial velocity excitation separately $\left(v(z)=0.1 \sin \left(\frac{\pi z}{10.045}\right)(n m / s)\right.$ in the $x$-axis and $v(z)=0.15 \sin \left(\frac{\pi z}{10.045}\right)(\mathrm{nm} / \mathrm{s})$ in the $y$-axis). Thus, in the first comparative test, we impose the initial velocity excitation in Eq. 12 on this Fe nanowire.

$$
\mathbf{v}(z)=\left[0.15 \sin \left(\frac{\pi z}{10.045}\right), 0.1 \sin \left(\frac{2 \pi z}{10.045}\right)\right]^{\mathrm{T}}(x-\text { axis, } y-\text { axis })
$$

The external energy versus time curve can be obtained from the simulation. As shown in Fig. 12a, a periodic pulsation pattern can be explicitly identified. This observation signifies that the beat phenomenon exists in this situation.

Table 1. Comparative tests between the Fe nanowire with similar natural frequencies and the $\mathrm{Fe}$ nanowire with similar excitation frequencies. 
Natural frequency

$$
\begin{aligned}
& v(z)=0.1 \sin \left(\frac{\pi z}{L}\right)(n m / s) \quad(x-\text { axis }) \quad 70.80 G H z \quad(x-\text { axis }) \quad 88.27 G H z \quad(x-\text { axis }) \\
& v(z)=0.15 \sin \left(\frac{\pi z}{L}\right)(n m / s) \quad(y-a x i s) \quad 30.83 G H z \quad(y-\text { axis }) \quad 89.72 G H z \quad(y-\text { axis })
\end{aligned}
$$

Excitation frequency

$\begin{array}{llllll}v(z)=0.1 \sin \left(\frac{\pi z}{L}\right)(\mathrm{nm} / \mathrm{s}) & (x-\text { axis }) & 70.80 G H z & (x-\text { axis }) & 88.27 G H z & (x-\text { axis }) \\ v(z)=0.1 \sin \left(\frac{2 \pi z}{L}\right)(\mathrm{nm} / \mathrm{s}) & (y-\text { axis }) & 74.31 \mathrm{GHz} & (y-\text { axis }) & 210.19 G H z & (y-\text { axis })\end{array}$

Initial velocity excitation of the comparative tests

$$
\mathbf{v}(z)=\left(\begin{array}{c}
0.15 \sin \left(\frac{\pi z}{10.045}\right) \\
0.1 \sin \left(\frac{2 \pi z}{10.045}\right)
\end{array}\right)(\mathrm{nm} / \mathrm{s}) \quad \mathbf{v}(z)=\left(\begin{array}{c}
0.1 \sin \left(\frac{\pi z}{8.61}\right) \\
0.15 \sin \left(\frac{2 \pi z}{8.61}\right)
\end{array}\right)(\mathrm{nm} / \mathrm{s})
$$

Then, we move to another simulation that is carried out on the Fe nanowire with the size of $1.722 \times 1.722 \times 8.61 \mathrm{~nm}^{3}$. Under different initial actuations $\left(v(z)=0.1 \sin \left(\frac{\pi z}{8.61}\right)(\mathrm{nm} / \mathrm{s})\right.$ in the $x$-axis and $v(z)=0.15 \sin \left(\frac{\pi z}{8.61}\right)(\mathrm{nm} / \mathrm{s})$ in the $y$-axis $)$, this nanowire has the similar natural frequencies (88.27 GHz and $89.72 \mathrm{GHz}$ ). When the initial velocity excitations $\left(v(z)=0.1 \sin \left(\frac{\pi z}{8.61}\right)(n m / s)\right.$ in the $x$-axis and $v(z)=0.1 \sin \left(\frac{2 \pi z}{8.61}\right)(n m / s)$ in the $y$-axis $)$ are imposed on it respectively, it has the different excitation frequencies (88.27 and $210.19 \mathrm{GHz})$. In this simulation, similarly, we impose the initial velocity excitation in Eq. 13 on this $\mathrm{Fe}$ nanowire.

$$
\mathbf{v}(z)=\left[0.1 \sin \left(\frac{\pi z}{8.61}\right), 0.15 \sin \left(\frac{2 \pi z}{8.61}\right)\right]^{\mathrm{T}}(x-\text { axis, } y-\text { axis })
$$

Fig. $12 \mathrm{~b}$ depicts the time history of the external energy. No distinguishing features could be found in Fig. 12b, so there is no evidence to show the existence of the beat phenomenon. Therefore, it is strongly confirmed that the beat phenomenon is excited by the vibrations with almost the same excitation frequency in two different directions. 

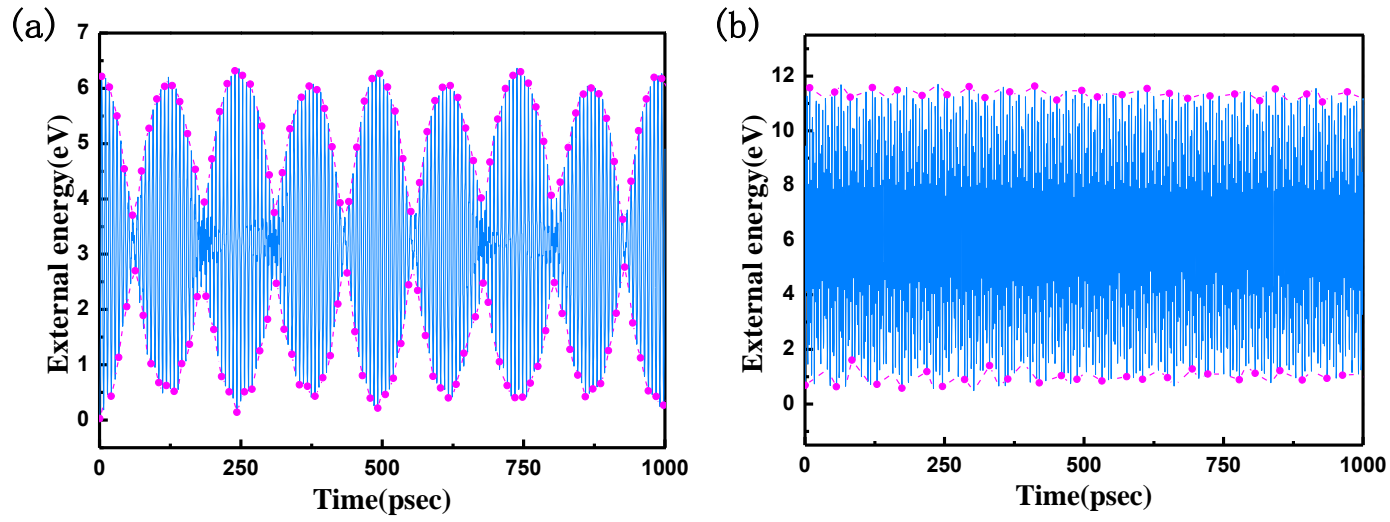

Figure 12. The results of comparative tests by MD simulations. They are the external energy time histories of the $\langle 100\rangle$ orientated Fe nanowires with the size of (a) $2.009 \times 0.574 \times 10.045 \mathrm{~nm}^{3}$ (b) $1.722 \times 1.722 \times 8.61 \mathrm{~nm}^{3}$. We can clearly see there are periodic pulsation patterns in (a), while no distinguishing features in (b).

\section{Conclusion}

In summary, based on large scale molecular dynamics simulations, we investigated beat phenomenon in the vibration of $\langle 100\rangle$ orientated $\mathrm{Fe}$ nanowires. It is found the beat phenomenon exists not only for $\langle 110\rangle$ orientated FCC nanowires of different materials $(\mathrm{Cu}$, $\mathrm{Au}, \mathrm{Ni}, \mathrm{Pd}$ and $\mathrm{Pt}$ ), but also for $<100>$ orientated $\mathrm{BCC}$ nanowires $(\mathrm{Fe})$. A discrete spring-mass model abstracted from the atomic arrangement is developed in this work to explore the displacement characteristics of beat phenomenon. With the increase of initial actuation amplitude, the vibration frequency of nanowires grows slowly. We chose an atom of middle cross-sectional (001) plane to reflect the deflection of the nanowire. Both the numerical results of the spring-mass model and the displacements of the typical atom in the nanowire reveal that the vibrations of the two elementary directions influence each other, which is the dynamical characteristics of beat phenomenon. In addition, the beat phenomenon driven by a single actuation along one of the elementary directions has also been observed. By a series of simulations, it is suggested the existence of plastic deformation leads to the decomposing of the single vibration along one of elementary directions, which further results in the appearance of beat phenomenon. Furthermore, the simulations have revealed the connection between the excitation mechanism of beat phenomenon and the frequency type. It is found that the excitation of beat phenomenon is related to the excitation frequency of the two elementary directions, instead of the natural frequency. 


\section{Appendix}

This spring-mass system consists of linear springs, which are defined as

$$
m \ddot{\mathbf{x}}=-k \Delta \mathbf{x}
$$

where $m$ is mass of the object, $k$ is elastic constant of the spring. Due to the symmetric cross-sectional (001) plane, the springs are chosen to be the same. $l_{0}$ is initial length of the spring.

For one-dimensional vibration (the object only vibrate along the $x$-axis), we analyze the forces of each spring on the object.

Spring (1) to the object:

$$
F_{1}=-k\left[l_{0}-\left(l_{0}-x\right)\right]
$$

Spring (2) to the object:

$$
F_{2}=-k\left[\left(l_{0}+x\right)-l_{0}\right]
$$

Spring (3) to the object:

$$
F_{3}=-k\left(\sqrt{l_{0}^{2}+x^{2}}-l_{0}\right) \frac{x}{\sqrt{l_{0}^{2}+x^{2}}}
$$

Spring (4) to the object:

$$
F_{4}=-k\left(\sqrt{l_{0}^{2}+x^{2}}-l_{0}\right) \frac{x}{\sqrt{l_{0}^{2}+x^{2}}}
$$

Hence, the dynamic equation of the spring-mass system for one-dimensional vibration is described as

$$
m \ddot{x}=-4 k x+2 k x \frac{l_{0}}{\sqrt{x^{2}+l_{0}^{2}}}
$$

In Eq. 6, it is obvious that the second item on the right is a nonlinear item for this equation. As shown in Fig. 3c, when this system vibrates along the $x$-axis, because of the spring-mass model's structure, the red springs produce the nonlinear forces, which correspond to the nonlinear item in Eq. 6. This is what we call the geometric nonlinearity.

For two-dimensional vibration(the object can vibrate along both the $x$-axis and $y$-axis), we analyze the forces of each spring on the object.

Spring (1) to the object: 


$$
\mathbf{F}_{1}=\left[-k\left(\sqrt{\left(x-l_{0}\right)^{2}+y^{2}}-l_{0}\right) \frac{x-l_{0}}{\sqrt{\left(x-l_{0}\right)^{2}+y^{2}}},-k\left(\sqrt{\left(x-l_{0}\right)^{2}+y^{2}}-l_{0}\right) \frac{y}{\sqrt{\left(x-l_{0}\right)^{2}+y^{2}}}\right]^{\mathrm{T}}
$$

Spring (2) to the object:

$$
\mathbf{F}_{2}=\left[-k\left(\sqrt{\left(x+l_{0}\right)^{2}+y^{2}}-l_{0}\right) \frac{x+l_{0}}{\sqrt{\left(x+l_{0}\right)^{2}+y^{2}}},-k\left(\sqrt{\left(x+l_{0}\right)^{2}+y^{2}}-l_{0}\right) \frac{y}{\sqrt{\left(x+l_{0}\right)^{2}+y^{2}}}\right]^{\mathrm{T}}
$$

Spring (3) to the object:

$$
\mathbf{F}_{3}=\left[-k\left(\sqrt{x^{2}+\left(y+l_{0}\right)^{2}}-l_{0}\right) \frac{x}{\sqrt{x^{2}+\left(y+l_{0}\right)^{2}}},-k\left(\sqrt{x^{2}+\left(y+l_{0}\right)^{2}}-l_{0}\right) \frac{y+l_{0}}{\sqrt{x^{2}+\left(y+l_{0}\right)^{2}}}\right]^{\mathrm{T}}
$$

Spring (4) to the object:

$$
\mathbf{F}_{4}=\left[-k\left(\sqrt{x^{2}+\left(y-l_{0}\right)^{2}}-l_{0}\right) \frac{x}{\sqrt{x^{2}+\left(y-l_{0}\right)^{2}}},-k\left(\sqrt{x^{2}+\left(y-l_{0}\right)^{2}}-l_{0}\right) \frac{y-l_{0}}{\sqrt{x^{2}+\left(y-l_{0}\right)^{2}}}\right]^{\mathrm{T}}
$$

Hence, the dynamic equations of this spring-mass system are described as

$$
\left\{\begin{array}{l}
m \ddot{x}=-4 k x-k l_{0}\left(\frac{-x}{\sqrt{x^{2}+\left(y-l_{0}\right)^{2}}}+\frac{-x}{\sqrt{x^{2}+\left(y+l_{0}\right)^{2}}}+\frac{-l_{0}-x}{\sqrt{\left(x+l_{0}\right)^{2}+y^{2}}}+\frac{l_{0}-x}{\sqrt{\left(x-l_{0}\right)^{2}+y^{2}}}\right) \\
m \ddot{y}=-4 k y-k l_{0}\left(\frac{l_{0}-y}{\sqrt{x^{2}+\left(y-l_{0}\right)^{2}}}+\frac{-l_{0}-y}{\sqrt{x^{2}+\left(y+l_{0}\right)^{2}}}+\frac{-y}{\sqrt{\left(x+l_{0}\right)^{2}+y^{2}}}+\frac{-y}{\sqrt{\left(x-l_{0}\right)^{2}+y^{2}}}\right)
\end{array}\right.
$$

Eq. 11 shows that there are several nonlinear items in these equations, while Fig. 3d gives the illustration of the two-dimensional vibration. When the object vibrates in both $x$-axis and $y$-axis, these nonlinear items with strong-coupling characteristics make this system more complicated.

\section{Supplementary Material}

See supplementary material for the video of the beat phenomenon in $\langle 100\rangle$ orientated Fe nanowire.

\section{Acknowledgements}

This work was supported in part by The National Natural Science Foundation of China (NSFC) under grant number 11072086, 11372117, J1310022, by the 985 program, by the 211 project of Jilin University, and by Basic funds for science and research in Jilin University. This work was also supported by the Jilin Province Computing Centre through the use of its high-performance computing facilities. 


\section{References}

[1] D. Rugar, R. Budakian, H.J. Mamin, B.W. Chui, Single spin detection by magnetic resonance force microscopy, Nature, 430 (2004) 329-332.

[2] T. Stowe, K. Yasumura, T. Kenny, D. Botkin, K. Wago, D. Rugar, Attonewton force detection using ultrathin silicon cantilevers, Applied Physics Letters, 71 (1997) 288-290.

[3] P. Xie, Q. Xiong, Y. Fang, Q. Qing, C.M. Lieber, Local electrical potential detection of DNA by nanowire-nanopore sensors, Nature nanotechnology, 7 (2012) 119-125.

[4] R.-M. Ma, L. Dai, H.-B. Huo, W.-J. Xu, G. Qin, High-performance logic circuits constructed on single CdS nanowires, Nano letters, 7 (2007) 3300-3304.

[5] C.K. Chan, H.L. Peng, G. Liu, K. Mcllwrath, X.F. Zhang, R.A. Huggins, Y. Cui, High-performance lithium battery anodes using silicon nanowires, Nature Nanotechnology, 3 (2008) 31-35.

[6] E. Gil-Santos, D. Ramos, J. Martínez, M. Fernández-Regúlez, R. García, Á. San Paulo, M. Calleja, J. Tamayo, Nanomechanical mass sensing and stiffness spectrometry based on two-dimensional vibrations of resonant nanowires, Nature nanotechnology, 5 (2010) 641-645.

[7] M. Li, T.S. Mayer, J.A. Sioss, C.D. Keating, R.B. Bhiladvala, Template-grown metal nanowires as resonators: Performance and characterization of dissipative and elastic properties, Nano Letters, 7 (2007) 3281-3284.

[8] X.-Q. Zeng, Y.-L. Wang, H. Deng, M.L. Latimer, Z.-L. Xiao, J. Pearson, T. Xu, H.-H. Wang, U. Welp, G.W. Crabtree, Networks of ultrasmall Pd/Cr nanowires as high performance hydrogen sensors, Acs Nano, 5 (2011) 7443-7452.

[9] Iron Nanorods, in, American elements, https://www.americanelements.com/iron-nanorods-7439-89-6.

[10] J. Martın, J. Nogues, K. Liu, J. Vicent, I.K. Schuller, Ordered magnetic nanostructures: fabrication and properties, Journal of magnetism and magnetic materials, 256 (2003) 449-501.

[11] G. Sainath, B. Choudhary, Orientation dependent deformation behaviour of BCC iron nanowires, Computational Materials Science, 111 (2016) 406-415.

[12] G. Sainath, B. Choudhary, Molecular dynamics simulations on size dependent tensile deformation behaviour of [110] oriented body centred cubic iron nanowires, Materials Science and Engineering: $A$, 640 (2015) 98-105.

[13] G. Sainath, B. Choudhary, T. Jayakumar, Molecular dynamics simulation studies on the size dependent tensile deformation and fracture behaviour of body centred cubic iron nanowires, Computational Materials Science, 104 (2015) 76-83.

[14] S.Y. Kim, H.S. Park, Utilizing mechanical strain to mitigate the intrinsic loss mechanisms in oscillating metal nanowires, Physical review letters, 101 (2008) 215502.

[15] X. Feng, R. He, P. Yang, M. Roukes, Very high frequency silicon nanowire electromechanical resonators, Nano Letters, 7 (2007) 1953-1959.

[16] H.S. Park, W. Cai, H.D. Espinosa, H. Huang, Mechanics of crystalline nanowires, MRS bulletin, 34 (2009) 178-183.

[17] S.S. Verbridge, J.M. Parpia, R.B. Reichenbach, L.M. Bellan, H. Craighead, High quality factor resonance at room temperature with nanostrings under high tensile stress, J Appl Phys, 99 (2006) 124304.

[18] Y. Yang, C. Callegari, X. Feng, M. Roukes, Surface adsorbate fluctuations and noise in nanoelectromechanical systems, Nano letters, 11 (2011) 1753-1759. 
[19] W.G. Conley, A. Raman, C.M. Krousgrill, S. Mohammadi, Nonlinear and nonplanar dynamics of suspended nanotube and nanowire resonators, Nano letters, 8 (2008) 1590-1595.

[20] J. He, C.M. Lilley, Surface stress effect on bending resonance of nanowires with different boundary conditions, Applied physics letters, 93 (2008) 263108.

[21] H.S. Park, Surface stress effects on the resonant properties of silicon nanowires, J Appl Phys, 103 (2008) 123504.

[22] H.F. Zhan, Y.T. Gu, A fundamental numerical and theoretical study for the vibrational properties of nanowires, J Appl Phys, 111 (2012).

[23] H.F. Zhan, Y.T. Gu, H.S. Park, Beat phenomena in metal nanowires, and their implications for resonance-based elastic property measurements, Nanoscale, 4 (2012) 6779-6785.

[24] J. Zhang, C. Wang, Beat vibration of hybrid boron nitride-carbon nanotubes-A new avenue to atomic-scale mass sensing, Computational Materials Science, 127 (2017) 270-276.

[25] P.A. Olsson, H.S. Park, P.C. Lidström, The Influence of shearing and rotary inertia on the resonant properties of gold nanowires, J Appl Phys, 108 (2010) 104312.

[26] S. Plimpton, Fast parallel algorithms for short-range molecular dynamics, Journal of computational physics, 117 (1995) 1-19.

[27] M.I. Mendelev, S. Han, D.J. Srolovitz, G.J. Ackland, D.Y. Sun, M. Asta, Development of new interatomic potentials appropriate for crystalline and liquid iron, Philos Mag, 83 (2003) 3977-3994.

[28] M.T. McDowell, A.M. Leach, K. Gall, Bending and tensile deformation of metallic nanowires, Model Simul Mater Sc, 16 (2008).

[29] C.L. Kelchner, S. Plimpton, J. Hamilton, Dislocation nucleation and defect structure during surface indentation, Physical review B, 58 (1998) 11085.

[30] W.G. Hoover, Canonical dynamics: equilibrium phase-space distributions, Physical review A, 31 (1985) 1695.

[31] S. Nosé, A unified formulation of the constant temperature molecular dynamics methods, The Journal of chemical physics, 81 (1984) 511-519. 